\title{
A FORMULA FOR THE $R$-MATRIX USING A SYSTEM OF WEIGHT PRESERVING ENDOMORPHISMS
}

\author{
PETER TINGLEY
}

\begin{abstract}
We give a formula for the universal $R$-matrix of the quantized universal enveloping algebra $U_{q}(\mathfrak{g})$. This is similar to a previous formula due to Kirillov-Reshetikhin and Levendorskii-Soibelman, except that where they use the action of the braid group element $T_{w_{0}}$ on each representation $V$, we show that one can instead use a system of weight preserving endomorphisms. One advantage of our construction is that it is well defined for all symmetrizable Kac-Moody algebras. However, we have only established that the result is equal to the universal $R$-matrix in finite type.
\end{abstract}

\section{INTRODUCTION}

Let $\mathfrak{g}$ be a finite type complex simple Lie algebra and $U_{q}(\mathfrak{g})$ the corresponding quantized universal enveloping algebra. In $[\mathrm{KR}]$ and $[\mathrm{LS}$, Kirillov-Reshetikhin and Levendorskii-Soibelman developed a formula for the universal $R$-matrix

$$
R=\left(X^{-1} \otimes X^{-1}\right) \Delta(X),
$$

where $X$ belongs to a completion of $U_{q}(\mathfrak{g})$. The element $X$ is constructed using the braid group element $T_{w_{0}}$ corresponding to the longest word of the braid group, and as such only makes sense when $\mathfrak{g}$ is of finite type.

The element $X$ in (1) defines a vector space endomorphism $X_{V}$ on each representation $V$ of $U_{q}(\mathfrak{g})$, and in fact $X$ is defined by the system of endomorphisms $\left\{X_{V}\right\}$. Furthermore, any natural system of vector space endomorphisms $\left\{E_{V}\right\}$ can be represented as an element $E$ in a certain completion of $U_{q}(\mathfrak{g})$ (see [KT]). The action of the coproduct $\Delta(E)$ on a tensor product $V \otimes W$ is then simply $E_{V \otimes W}$. Thus the right side of (11) is well defined if $X$ is replaced by $E=\left\{E_{V}\right\}$.

In this note we consider the case where $\mathfrak{g}$ is a symmetrizable Kac-Moody algebra. We define a system of weight preserving endomorphisms $\Theta=\left\{\Theta_{V}\right\}$ of all integrable highest weight representations $V$ of $U_{q}(\mathfrak{g})$. When $\mathfrak{g}$ is of finite type, we show that

$$
R=\left(\Theta^{-1} \otimes \Theta^{-1}\right) \Delta(\Theta),
$$

where the equality means that, for any type $\mathbf{1}$ finite dimensional modules $V$ and $W$, the actions of the two sides of (2) on $V \otimes W$ agree. We expect this remains true in other cases, although this has not been proven.

Our endomorphisms $\Theta_{V}$ are not linear over the field $\mathbb{C}(q)$, but are instead compatible with the automorphism which inverts $q$. For this reason, $\Theta$ cannot be realized using an element in a completion of $U_{q}(\mathfrak{g})$, and it is crucial to work with

Received by the editors February 24, 2008.

2010 Mathematics Subject Classification. Primary 17B37; Secondary 16Txx.

This work was supported by the RTG grant DMS-0354321.

(C)2010 American Mathematical Society Reverts to public domain 28 years from publication 
systems of endomorphisms. There is a further technicality in that $\Theta_{V}$ actually depends on a choice of global basis for $V$. Nonetheless, we give a precise meaning to (2).

This note is organized as follows. In Section 2 we fix notation and conventions. In Section 3 we review the universal $R$-matrix. In Section 4 we review a method developed by Henriques and Kamnitzer [HK] to construct isomorphisms $V \otimes W \rightarrow$ $W \otimes V$. In Section [5 we state some background results on crystal bases and global bases. In Section 6 we construct our endomorphism $\Theta$. In Section 7 we prove our main theorem (Theorem 7.11), which establishes (2) when $\mathfrak{g}$ is of finite type. In Section 8 we briefly discuss future directions for this work.

\section{Conventions}

We must first fix some notation. For the most part we follow $\mathrm{CP}$.

- $\mathfrak{g}$ is a symmetrizable Kac-Moody algebra with Cartan matrix $A=\left(a_{i j}\right)_{i, j \in I}$ and Cartan subalgebra $\mathfrak{h}$.

$\bullet\langle\cdot, \cdot\rangle$ denotes the paring between $\mathfrak{h}$ and $\mathfrak{h}^{\star}$ and $(\cdot, \cdot)$ denotes the usual symmetric bilinear form on either $\mathfrak{h}$ or $\mathfrak{h}^{\star}$. Fix the usual elements $\alpha_{i} \in \mathfrak{h}^{\star}$ and $H_{i} \in \mathfrak{h}$, and recall that $\left\langle H_{i}, \alpha_{j}\right\rangle=a_{i j}$.

- $d_{i}=\left(\alpha_{i}, \alpha_{i}\right) / 2$, so that $\left(H_{i}, H_{j}\right)=d_{j}^{-1} a_{i j}$ and, for all $\lambda \in \mathfrak{h}^{*},\left(\alpha_{i}, \lambda\right)=$ $d_{i}\left\langle H_{i}, \lambda\right\rangle$.

- $B$ is the symmetric matrix $\left(d_{j}^{-1} a_{i j}\right)$.

- $\rho \in \mathfrak{h}^{*}$ satisfies $\left\langle H_{i}, \rho\right\rangle=1$ for all $i$. Note that this implies $\left(\alpha_{i}, \rho\right)=d_{i}$. If $A$ is not invertible this condition does not uniquely determine $\rho$, and we simply choose any one solution.

- $H_{\rho}$ is the element of $\mathfrak{h}$ such that, for any $\lambda \in \mathfrak{h}^{*},\left\langle H_{\rho}, \lambda\right\rangle=(\rho, \lambda)$. In particular, $\left\langle H_{\rho}, \alpha_{i}\right\rangle=d_{i}$ for all $i$.

- $U_{q}(\mathfrak{g})$ is the quantized universal enveloping algebra associated to $\mathfrak{g}$, generated over $\mathbb{C}(q)$ by $E_{i}, F_{i}$ for all $i \in I$, and $K_{H}$ for $H$ in the coweight lattice of $\mathfrak{g}$. As usual, let $K_{i}=K_{d_{i} H_{i}}$. For convenience, we recall the exact formula for the coproduct

$$
\left\{\begin{array}{l}
\Delta E_{i}=E_{i} \otimes K_{i}+1 \otimes E_{i}, \\
\Delta F_{i}=F_{i} \otimes 1+K_{i}^{-1} \otimes F_{i}, \\
\Delta K_{H}=K_{H} \otimes K_{H},
\end{array}\right.
$$

and the commutation relations

$$
K_{H} E_{i} K_{H}^{-1}=q^{\left\langle H, \alpha_{i}\right\rangle} E_{i} \quad \text { and } \quad K_{H} F_{i} K_{H}^{-1}=q^{-\left\langle H, \alpha_{i}\right\rangle} F_{i} .
$$

At times it will be necessary to adjoin a fixed $k$ th root of $q$ to the base field $\mathbb{C}(q)$, where $k$ is twice the dual Coxeter number of $\mathfrak{g}$.

- $[n]=\frac{q^{n}-q^{-n}}{q-q^{-1}}$, and $X^{(n)}=\frac{X^{n}}{[n][n-1] \cdots[2]}$.

- Fix a representation $V$ of $U_{q}(\mathfrak{g})$ and $\lambda \in \mathfrak{h}^{*}$. We say $v \in V$ is a weight vector of weight $\lambda$ if, for all $H \in \mathfrak{h}, K_{H}(v)=q^{\langle H, \lambda\rangle} v$.

- $\lambda \in \mathfrak{h}^{*}$ is called a dominant integral weight if $\left\langle H_{i}, \lambda\right\rangle \in \mathbb{Z}_{\geq 0}$ for all $i$.

- For each dominant integral weight $\lambda, V_{\lambda}$ is the type $\mathbf{1}$ irreducible integrable representation of $U_{q}(\mathfrak{g})$ with highest weight $\lambda$.

- $B_{\lambda}$ is a fixed global basis for $V_{\lambda}$, in the sense of Kashiwara (see [K]). $b_{\lambda}$ and $b_{\lambda}^{\text {low }}$ are the highest weight and lowest weight elements of $B_{\lambda}$, respectively. 


\section{The $R$-MATRIX}

We briefly recall the definition of a universal $R$-matrix, and the related notion of a braiding.

Definition 3.1. A braided monoidal category is a monoidal category $\mathcal{C}$, along with a natural system of isomorphisms $\sigma_{V, W}^{b r}: V \otimes W \rightarrow W \otimes V$ for each pair $V, W \in \mathcal{C}$, such that, for any $U, V, W \in \mathcal{C}$, the following two equalities hold:

$$
\begin{aligned}
& \left(\sigma_{U, W}^{b r} \otimes \mathrm{Id}\right) \circ\left(\mathrm{Id} \otimes \sigma_{V, W}^{b r}\right)=\sigma_{U \otimes V, W}^{b r}, \\
& \left(\mathrm{Id} \otimes \sigma_{U, W}^{b r}\right) \circ\left(\sigma_{U, V}^{b r} \otimes \mathrm{Id}\right)=\sigma_{U, V \otimes W}^{b r} .
\end{aligned}
$$

The system $\sigma^{b r}:=\left\{\sigma_{V, W}^{b r}\right\}$ is called a braiding on $\mathcal{C}$.

Let $U_{q}\left(\widetilde{\mathfrak{g}) \otimes U_{q}}(\mathfrak{g})\right.$ be the completion of $U_{q}(\mathfrak{g}) \otimes U_{q}(\mathfrak{g})$ in the weak topology defined by all matrix elements of representations $V_{\lambda} \otimes V_{\mu}$, for all ordered pairs of dominant integral weights $(\lambda, \mu)$.

Definition 3.2. A universal $R$-matrix is an element $R$ of $U_{q}\left(\widetilde{\mathfrak{g}) \otimes U_{q}}(\mathfrak{g})\right.$ such that $\sigma_{V, W}^{b r}:=$ Flip $\circ R$ is a braiding on the category of $U_{q}(\mathfrak{g})$ representations.

Note in particular that, since the braiding is an isomorphism, $R$ must be invertible. It is central to the theory of quantized universal enveloping algebras that, for any symmetrizable Kac-Moody algebra $\mathfrak{g}, U_{q}(\mathfrak{g})$ has a universal $R$-matrix. The universal $R$-matrix is not truly unique, but there is a well-studied standard choice. See $[\mathrm{CP}]$ for a thorough discussion when $\mathfrak{g}$ is of finite type, and $[\mathrm{L}$ for the general case.

When $\mathfrak{g}$ is of finite type, the $R$-matrix can be described explicitly as follows. Note that the expression below is presented in the $h$-adic completion of $U_{h}(\mathfrak{g})$, whereas here we are working in $U_{q}(\mathfrak{g})$. However, it is straightforward to check that this gives a well-defined endomorphism of $V \otimes W$ for any integrable highest weight $U_{q}(\mathfrak{g})$-representations $V$ and $W$, with the only difficulty being that certain fractional powers of $q$ can appear.

Theorem 3.3 (see $[\mathrm{CP}$, Theorem 8.3.9]). Assume $\mathfrak{g}$ is of finite type. Then the standard universal $R$-matrix for $U_{q}(\mathfrak{g})$ is given by the expression

$$
R_{h}=\exp \left(h \sum_{i, j}\left(B^{-1}\right)_{i j} H_{i} \otimes H_{j}\right) \prod_{\beta} \exp _{q_{\beta}}\left[\left(1-q_{\beta}^{-2}\right) E_{\beta} \otimes F_{\beta}\right],
$$

where the product is over all the positive roots of $\mathfrak{g}$, and the order of the terms is such that $\beta_{r}$ appears to the left of $\beta_{s}$ if $r>s$.

We will not explain all of the notation in ([6), since the only thing we use is the fact that $E_{\beta}$ acts as 0 on any highest weight vector, and so the product in the expression acts as the identity on $b_{\lambda} \otimes c \in V_{\lambda} \otimes V_{\mu}$.

\section{Constructing isomorphisms Using SYSTEMS OF ENDOMORPHiSMS}

Here and throughout this note a representation of $U_{q}(\mathfrak{g})$ will mean a direct sum of possibly infinitely many of the irreducible integrable type 1 representations $V_{\lambda}$. We note that the category of such representations is closed under the tensor product. When $\mathfrak{g}$ is of finite type, we can restrict to finite direct sums, or equivalently finite 
dimensional type $\mathbf{1}$ modules, since this category is already closed under the tensor product.

In this section we review a method for constructing natural systems of isomorphisms $\sigma_{V, W}: V \otimes W \rightarrow W \otimes V$. This idea was used by Henriques and Kamnitzer in $[\mathrm{HK}$, and was further developed in [KT]. The data needed to construct such a system is:

(i) an algebra automorphism $C_{\xi}$ of $U_{q}(\mathfrak{g})$ which is also a coalgebra antiautomorphism;

(ii) a natural system of invertible vector space endomorphisms $\xi_{V}$ of each representation $V$ of $U_{q}(\mathfrak{g})$ which is compatible with $C_{\xi}$ in the sense that the following diagram commutes for all $V$ :

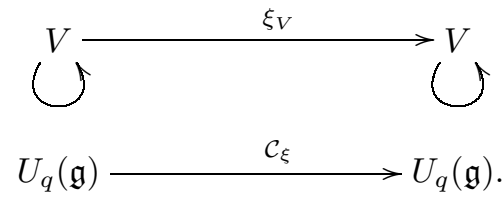

It follows immediately from the definition of coalgebra anti-automorphism that

$$
\sigma_{V, W}^{\xi}:=\operatorname{Flip} \circ\left(\xi_{V}^{-1} \otimes \xi_{W}^{-1}\right) \circ \xi_{V \otimes W}
$$

is an isomorphism of $U_{q}(\mathfrak{g})$ representations from $V \otimes W$ to $W \otimes V$, where Flip is the map from $V \otimes W$ to $W \otimes V$ defined by $\operatorname{Flip}(v \otimes w)=w \otimes v$.

We will normally denote the system $\left\{\xi_{V}\right\}$ simply by $\xi$, and we will denote the action of $\xi$ on the tensor product of two representations by $\Delta(\xi)$. This is justified since, as explained in [KT], $\xi$ in fact belongs to a completion of $U_{q}(\mathfrak{g})$, and the action of $\xi$ on $V \otimes W$ is calculated using the coproduct. With this notation $\sigma^{\xi}:=\left\{\sigma_{V, W}^{\xi}\right\}$ can be expressed as

$$
\sigma^{\xi}=\text { Flip } \circ\left(\xi^{-1} \otimes \xi^{-1}\right) \circ \Delta(\xi) .
$$

In the current work we require a little more freedom: we will sometimes use automorphisms $C_{\xi}$ of $U_{q}(\mathfrak{g})$ which are not linear over $\mathbb{C}(q)$, but instead are barlinear (i.e. invert $q$ ). This causes some technical difficulties, which we deal with in Section 6. Once we make this precise, we will use all the same notation for a bar-linear $C_{\xi}$ and compatible system of $\mathbb{C}$ vector space automorphisms $\xi$ as we do in the linear case, including using $\Delta(\xi)$ to denote $\xi$ acting on a tensor product.

Comment 4.1. Since the representations we are considering are all completely reducible, to describe the data $\left(\mathcal{C}_{\xi}, \xi\right)$ it is sufficient to describe $C_{\xi}$ and to give the action of $\xi_{V_{\lambda}}$ on any one vector $v$ in each irreducible representation $V_{\lambda}$. This is usually more convenient than describing $\xi_{V_{\lambda}}$ explicitly. Of course, the choice of $C_{\xi}$ imposes a restriction on $\xi_{V_{\lambda}}(v)$, so when we give such a description of $\xi$, we must check that the action on our chosen vector in each $V_{\lambda}$ is compatible with $C_{\xi}$.

Comment 4.2. If $C_{\xi}$ is an coalgebra automorphism as opposed to a coalgebra antiautomorphism, the same arguments show that $\left(\xi_{V}^{-1} \otimes \xi_{W}^{-1}\right) \circ \xi_{V \otimes W}: V \otimes W \rightarrow V \otimes W$ is an isomorphism.

\section{Crystal Bases and GLOBAl BASES}

In order to extend the construction described in Section 4 to include bar-linear $\xi$, we will need to use some results concerning crystal bases and global bases. We 
state only what is relevant to us, and refer the reader to $[\mathrm{K}$ for a more complete exposition. Unfortunately, the conventions in $[\mathrm{K}]$ and $[\mathrm{CP}$ do not quite agree. In particular, the theorems from $[\mathrm{K}]$ that we will need are stated in terms of a different coproduct, so we have modified them to match our conventions.

Definition 5.1. Fix an integrable highest weight representation $V$ of $U_{q}(\mathfrak{g})$. Define the Kashiwara operators $\tilde{F}_{i}, \tilde{E}_{i}: V \rightarrow V$ by linearly extending

$$
\left\{\begin{array}{l}
\tilde{F}_{i}\left(F_{i}^{(n)}(v)\right)=F_{i}^{(n+1)}(v), \\
\tilde{E}_{i}\left(F_{i}^{(n)}(v)\right)=F_{i}^{(n-1)}(v) .
\end{array}\right.
$$

for all $v \in V$ such that $E_{i}(v)=0$.

Definition 5.2. Let $\mathcal{A}_{\infty}=\mathbb{C}\left[q^{-1}\right]_{0}$ be the algebra of rational functions in $q^{-1}$ over $\mathbb{C}$ whose denominators are not divisible by $q^{-1}$.

Definition 5.3. A crystal basis of a representation $V($ at $q=\infty)$ is a pair $(\mathcal{L}, \widetilde{B})$, where $\mathcal{L}$ is an $\mathcal{A}_{\infty}$-lattice of $V$ and $\widetilde{B}$ is a basis for $\mathcal{L} / q^{-1} \mathcal{L}$, such that:

(i) $\mathcal{L}$ and $\widetilde{B}$ are compatible with the weight decomposition of $V$.

(ii) $\mathcal{L}$ is invariant under the Kashiwara operators and $\widetilde{B} \cup 0$ is invariant under their residues $e_{i}:=\tilde{E}_{i}^{\left(\bmod q^{-1} \mathcal{L}\right)}, f_{i}:=\tilde{F}_{i}^{\left(\bmod q^{-1} \mathcal{L}\right)}: \mathcal{L} / q^{-1} \mathcal{L} \rightarrow \mathcal{L} / q^{-1} \mathcal{L}$.

(iii) For any $b, b^{\prime} \in \widetilde{B}$, we have $e_{i} b=b^{\prime}$ if and only if $f_{i} b^{\prime}=b$.

Definition 5.4. Let $(\mathcal{L}, \widetilde{B})$ be a crystal basis for $V$. The highest weight elements of $\widetilde{B}$ are those $b \in \widetilde{B}$ such that, for all $i, e_{i}(b)=0$.

Proposition 5.5 (see $[\mathrm{K}]$ ). Each $V_{\lambda}$ has a crystal basis $\left(\mathcal{L}_{\lambda}, \widetilde{B}_{\lambda}\right)$. Furthermore, $\left(\mathcal{L}_{\lambda}, \widetilde{B}_{\lambda}\right)$ has a unique highest weight element, and this occurs in the $\lambda$ weight space.

Theorem 5.6 ([K, Theorem 1]). Let $V, W$ be representations with crystal bases $(\mathcal{L}, \widetilde{A})$ and $(\mathcal{M}, \widetilde{B})$, respectively. Then $(\mathcal{L} \otimes \mathcal{M}, \widetilde{A} \otimes \widetilde{B})$ is a crystal basis of $V \otimes W$. Furthermore, the highest weight elements of $\widetilde{A} \otimes \widetilde{B}$ are all of the form $a^{\text {high }} \otimes b$, where $a^{\text {high }}$ is a highest weight element of $\widetilde{A}$.

Definition 5.7. Let $\left(\mathcal{L}_{\lambda}, \widetilde{B}_{\lambda}\right)$ and $\left(\mathcal{L}_{\mu}, \widetilde{B}_{\mu}\right)$ be crystal bases for $V_{\lambda}$ and $V_{\mu}$. Set

$$
S_{\lambda, \mu}^{\nu}:=\left\{b \in \widetilde{B}_{\mu}: b_{\lambda} \otimes b \text { is a highest weight element of } \widetilde{B}_{\lambda} \otimes \widetilde{B}_{\mu} \text { of weight } \nu\right\} \text {. }
$$

For any $V_{\lambda}$, and any choice of highest weight vector $b_{\lambda} \in V_{\lambda}$, there is a canonical choice of basis $B_{\lambda}$ for $V_{\lambda}$, which contains $b_{\lambda}$, and such that $\left(B_{\lambda}+q \mathcal{L}, \mathcal{L}\right)$ is a crystal basis for $V$, where $\mathcal{L}$ is the $\mathcal{A}_{\infty}$-span of $B_{\lambda}$. That is not to say there is a unique basis for $V_{\lambda}$ satisfying these two conditions, only that one can find a canonical "good" choice. This is known as the global basis for $V_{\lambda}$. A complete construction can be found in $[\mathrm{K}$, although here we more closely follow the presentation from CP. Chapter $14.1 \mathrm{C}]$. In the present work we simply use the fact that the global basis exists, and state the properties of $B_{\lambda}$ that we need.

Definition 5.8. $C_{\text {bar }}: U_{q}(\mathfrak{g}) \rightarrow U_{q}(\mathfrak{g})$ is the $\mathbb{C}$-algebra involution defined by

$$
\left\{\begin{array}{l}
C_{\mathrm{bar}}\left(E_{i}\right)=E_{i}, \\
C_{\mathrm{bar}}\left(F_{i}\right)=F_{i}, \\
C_{\mathrm{bar}}\left(K_{i}\right)=K_{i}^{-1}, \\
C_{\mathrm{bar}}(q)=q^{-1} .
\end{array}\right.
$$


Theorem 5.9 (Kashiwara $[\mathrm{K}]$ ). Fix a highest weight vector $b_{\lambda} \in V_{\lambda}$. There is a canonical choice of a "global" basis $B_{\lambda}$ of $V_{\lambda}$. This has the properties (although it is not defined by these alone) that:

(i) $b_{\lambda} \in B_{\lambda}$.

(ii) $B_{\lambda}$ is a weight basis for $V_{\lambda}$.

(iii) Let $\mathcal{L}$ be the $\mathcal{A}_{\infty}$ span of $B_{\lambda}$. Then $\left(B_{\lambda}+q^{-1} \mathcal{L}, \mathcal{L}\right)$ is a crystal basis for $V_{\lambda}$.

(iv) Define the involution $\operatorname{bar}_{\left(V_{\lambda}, B_{\lambda}\right)}$ of $V_{\lambda}$ by $\operatorname{bar}_{\left(V_{\lambda}, B_{\lambda}\right)}(f(q) b)=f\left(q^{-1}\right) b$ for all $f(q) \in \mathbb{C}(q)$ and $b \in B_{\lambda}$. Then $\operatorname{bar}_{\left(V_{\lambda}, B_{\lambda}\right)}$ is compatible with $C_{\mathrm{bar}}$, in the sense discussed in Section 4 .

Furthermore, if a different highest weight vector is chosen, $B_{\lambda}$ is multiplied by an overall scalar.

Definition 5.10. If $V$ is any (possibly reducible) representation of $U_{q}(\mathfrak{g})$, we say a basis $B$ of $V$ is a global basis if there is a decomposition of $V$ into irreducible components such that $B$ is a union of global bases for the irreducible pieces.

\section{The SYSTEM OF ENDOMORPhisms $\Theta$}

We now introduce a $\mathbb{C}$-algebra automorphism $C_{\Theta}$ of $U_{q}(\mathfrak{g})$. Notice that this inverts $q$, so it is not a $\mathbb{C}(q)$-algebra automorphism, but is instead bar-linear:

$$
\left\{\begin{array}{l}
C_{\Theta}\left(E_{i}\right)=E_{i} K_{i}^{-1}, \\
C_{\Theta}\left(F_{i}\right)=K_{i} F_{i}, \\
C_{\Theta}\left(K_{i}\right)=K_{i}^{-1}, \\
C_{\Theta}(q)=q^{-1} .
\end{array}\right.
$$

One can check that $C_{\Theta}$ is a well-defined algebra involution and a coalgebra antiinvolution. In order to use the methods of Section 4 , we must define a $\mathbb{C}$-vector space automorphism $\Theta_{V_{\lambda}}$ of each $V_{\lambda}$ which is compatible with $C_{\Theta}$. This is complicated by the fact that $C_{\Theta}$ does not preserve the $\mathbb{C}(q)$-algebra structure, but instead inverts $q$. We must actually work in the category of representations with chosen global bases. An element of this category will be denoted $(V, B)$, where $B$ is the chosen global basis of $V$.

Definition 6.1. Fix a global basis $B_{\lambda}$ for $V_{\lambda}$. The action of $\Theta_{\left(V_{\lambda}, B_{\lambda}\right)}$ on $V_{\lambda}$ is defined by requiring that it be compatible with $C_{\Theta}$, and that $\Theta_{\left(V_{\lambda}, B_{\lambda}\right)}\left(b_{\lambda}\right)=$ $q^{-(\lambda, \lambda) / 2+(\lambda, \rho)} b_{\lambda}$. This is extended by naturality to define $\Theta_{(V, B)}$ for any (possibly reducible) $V$.

Comment 6.2. To ensure that Definition 6.1 makes sense, one must check that there is a map which sends $b_{\lambda}$ to $q^{-(\lambda, \lambda) / 2+(\lambda, \rho)} b_{\lambda}$ and is compatible with $C_{\Theta}$. This amounts to checking that $b_{\lambda}$ is still a highest weight vector if the action of $U_{q}(\mathfrak{g})$ is twisted by the automorphism $C_{\Theta}$, and is not difficult.

Comment 6.3. In some cases $\Theta$ acts on a weight vector as multiplication by a fractional power of $q$. To be completely precise we should adjoin a fixed $k$ th root of unity to the base field $\mathbb{C}(q)$, where $k$ is twice the dual Coxeter number of $\mathfrak{g}$. This causes no significant difficulties.

The construction described in Section 4 uses the action of $\xi_{V \otimes W}$ on $V \otimes W$. Thus we will need to define how $\Theta$ acts on a tensor product. In particular, we 
need a well-defined notion of tensor product in the category of representations with chosen global bases.

Definition 6.4. Let $V_{\lambda, \mu}^{\nu}$ denote the isotypic component of $V_{\lambda} \otimes V_{\mu}$ with highest weight $\nu$. Let $V_{\lambda, \mu}^{>\nu}:=\bigcup_{\gamma>\nu} V_{\lambda, \mu}^{\gamma}, V_{\lambda, \mu}^{\geq \nu}:=\bigcup_{\gamma \geq \nu} V_{\lambda, \mu}^{\gamma}$, and $Q_{\lambda, \mu}^{\nu}:=V_{\lambda, \mu}^{\geq \nu} / V_{\lambda, \mu}^{>\nu}$. Here we use the partial order of the weight lattice where $\gamma \geq \nu$ iff $\gamma-\nu$ is a nonnegative linear combination of the $\alpha_{i}$.

Comment 6.5. It is clear that the inclusion $V_{\lambda, \mu}^{\nu} \hookrightarrow V_{\lambda, \mu}^{\geq \nu}$ descends to an isomorphism from $V_{\lambda, \mu}^{\nu}$ to $Q_{\lambda, \mu}^{\nu}$.

Definition 6.6. The tensor product $\left(V_{\lambda}, B_{\lambda}\right) \otimes\left(V_{\mu}, B_{\mu}\right)$ is defined to be $\left(V_{\lambda} \otimes\right.$ $\left.V_{\mu}, A\right)$, where $A$ is the unique global basis of $V \otimes W$ such that the projections of the highest weight elements of $A$ of weight $\nu$ in $Q_{\lambda, \mu}^{\nu}$ are equal to the projections of $b_{\lambda} \otimes b$ for those $b \in S_{\lambda, \mu}^{\nu}$. This is well defined by Comment 6.5. Extend by naturality a tensor product $(V, B) \otimes(W, C)$ for possibly reducible $V$ and $W$.

\section{Proof that we obtain the $R$-matrix When $\mathfrak{g}$ is of Finite type}

The proof of our main theorem uses a relationship between the $R$-matrix and the braid group element $T_{w_{0}}$ first observed in $[\mathrm{KR}$ and [LS]. Thus for this section we must restrict to a finite type. We hope the result will prove to be true in greater generality, but establishing this would certainly require a different approach. We start by introducing a few more automorphisms of $U_{q}(\mathfrak{g})$ and of its representations.

Definition 7.1. Let $\theta$ to be the diagram automorphism such that $w_{0}\left(\alpha_{i}\right)=-\alpha_{\theta(i)}$, where $w_{0}$ is the longest element in the Weyl group.

Definition 7.2. $C_{\Gamma}$ is the $\mathbb{C}$-Hopf algebra automorphism of $U_{q}(\mathfrak{g})$ defined by

$$
\left\{\begin{array}{l}
C_{\Gamma}\left(E_{i}\right)=-K_{\theta(i)} F_{\theta(i)}, \\
C_{\Gamma}\left(F_{i}\right)=-E_{\theta(i)} K_{\theta(i)}^{-1}, \\
C_{\Gamma}\left(K_{i}\right)=K_{\theta(i)}, \\
C_{\Gamma}(q)=q^{-1}
\end{array}\right.
$$

Define the action of $\Gamma_{\left(V_{\lambda}, B_{\lambda}\right)}$ on $V_{\lambda}$ to be the unique $\mathbb{C}$-linear endomorphism of each $V_{\lambda}$ which is compatible with $C_{\Gamma}$, and which is normalized so that $\Gamma\left(b_{\lambda}\right)=b_{\lambda}^{\text {low }}$. Extend this by naturality to get the action of $\Gamma_{(V, B)}$ on any (possibly reducible) representation $V$ with chosen global basis $B$.

Comment 7.3. It is a simple exercise to check that $C_{\Gamma}$ is in fact a Hopf algebra automorphism, and is compatible with a $\mathbb{C}$-vector space automorphism of $V_{\lambda}$ which takes $b_{\lambda}$ to $b_{\lambda}^{\text {low }}$.

Definition 7.4. $C_{T_{w_{0}}}$ and $C_{J}$ are the $\mathbb{C}(q)$-algebra automorphisms of $U_{q}(\mathfrak{g})$ defined by

$$
\begin{aligned}
& \left\{\begin{array}{l}
C_{T_{w_{0}}}\left(E_{i}\right)=-F_{\theta(i)} K_{\theta(i)}, \\
C_{T_{w_{0}}}\left(F_{i}\right)=-K_{\theta(i)}^{-1} E_{\theta(i)}, \\
C_{T_{w_{0}}}\left(K_{H}\right)=K_{w_{0}(H)}, \text { so that } C_{T_{w_{0}}}\left(K_{i}\right)=K_{\theta(i)}^{-1},
\end{array}\right. \\
& \left\{\begin{array}{l}
C_{J}\left(E_{i}\right)=K_{i} E_{i}, \\
C_{J}\left(F_{i}\right)=F_{i} K_{i}^{-1}, \\
C_{J}\left(K_{H}\right)=K_{H} .
\end{array}\right.
\end{aligned}
$$


The systems of $\mathbb{C}(q)$-vector space automorphisms $T_{w_{0}}$ and $J$ of each $V_{\lambda}$ are the unique automorphisms which are compatible with $C_{T_{w_{0}}}$ and $C_{J}$, respectively, and such that $T_{w_{0}}\left(b_{\lambda}^{\text {low }}\right)=b_{\lambda}$ and $J\left(b_{\lambda}\right)=q^{(\lambda, \lambda) / 2+(\lambda, \rho)} b_{\lambda}$, where $b_{\lambda}$ and $b_{\lambda}^{\text {low }}$ are the highest and lowest weight elements in some global basis $B_{\lambda}$.

Comment 7.5. It is straightforward exercise to show that the formulas in Definition 7.4 do define algebra automorphisms of $U_{q}(\mathfrak{g})$ and compatible vector space automorphisms of each $V_{\lambda}$. There is an action of the braid group on each $V_{\lambda}$, and $T_{w_{0}}$ is in fact the action of the longest element (for an appropriate choice of conventions). Note also that $J$ and $T_{w_{0}}$ do not depend on the choice of global basis as they are stable under simultaneously rescaling $b_{\lambda}$ and $b_{\lambda}^{\text {low }}$. All of this is discussed in $\mathrm{KT}$.

Lemma 7.6. The following identities hold:

(i) $\Gamma_{(V, B)}=\operatorname{bar}_{(V, B)} \circ T_{w_{0}}^{-1}$,

(ii) $\Theta_{(V, B)}=K_{2 H_{\rho}} \circ \operatorname{bar}_{(V, B)} \circ J$,

(iii) for any weight vector $v \in V$ with $\operatorname{wt}(v)=\mu, J(v)=q^{(\mu, \mu) / 2+(\mu, \rho)} v$,

(iv) for any $b \in B$ with $\operatorname{wt}(b)=\mu, \Theta_{(V, B)}(b)=q^{-(\mu, \mu) / 2+(\mu, \rho)} b$,

(v) $\Gamma_{(V, B)}^{-1} \circ \Theta_{(V, B)}=J T_{w_{0}}$.

Here $\operatorname{bar}_{(V, B)}$ is the involution defined in Theorem [5.9, part (iv).

Proof. Let $C_{K_{2 H_{\rho}}}$ be the algebra automorphism of $U_{q}(\mathfrak{g})$ defined by $C_{K_{2 H_{\rho}}}(X)=$ $K_{2 H_{\rho}} X K_{2 H_{\rho}}^{-1}$. It follows directly from (4) that

$$
C_{K_{2 H_{\rho}}}\left(K_{i}^{-1} E_{i}\right)=E_{i} K_{i}^{-1} \quad \text { and } C_{K_{2 H_{\rho}}}\left(F_{i} K_{i}\right)=K_{i} F_{i} \text {. }
$$

Using (15) and the relevant definitions, a simple check on generators shows that

$$
C_{\Gamma}=C_{\mathrm{bar}} \circ C_{T_{w_{0}}}^{-1}, C_{\Theta}=C_{K_{2 H_{\rho}}} \circ C_{\mathrm{bar}} \circ C_{J}, \text { and } C_{\Gamma}^{-1} \circ C_{\Theta}=C_{J} \circ C_{T_{w_{0}}} .
$$

Thus, to prove (ii), (iii) and (ㅍ) , it suffices to check each identity when each side acts on any one chosen vector $b$ in each $V_{\lambda}$. For parts (ii) and (iii), choose $b=b_{\lambda}$ and the identity is immediate from the definitions.

For part (iii), it is sufficient to consider $V=V_{\lambda}$. By Definition 7.4, (iii) holds for $b=b_{\lambda}$. Furthermore, vectors of the form $F_{i_{k}} \cdots F_{i_{1}} b_{\lambda}$ generate $V_{\lambda}$ as a $\mathbb{C}(q)$ module. Assume that $v$ is a weight vector of weight $\mu$, and $J(v)=q^{(\mu, \mu) / 2+(\mu, \rho)} v$. Fix $i \in I$. Then

$$
\begin{aligned}
J\left(F_{i} v\right) & =C_{J}\left(F_{i}\right) J(v)=F_{i} K_{i}^{-1} q^{(\mu, \mu) / 2+(\mu, \rho)} v=F_{i} q^{-\left\langle d_{i} H_{i}, \mu\right\rangle} q^{(\mu, \mu) / 2+(\mu, \rho)} v \\
& =q^{-\left(\alpha_{i}, \mu\right)} q^{(\mu, \mu) / 2+(\mu, \rho)} v=q^{\left(\mu-\alpha_{i}, \mu-\alpha_{i}\right) / 2+\left(\mu-\alpha_{i}, \rho\right)} v .
\end{aligned}
$$

The claim now follows by induction on $k$.

Part (iv) follows by directly calculating the action of the right side of (iii) on $b$ and using part (iii) to evaluate the action of $J$.

The definitions of $\Theta_{(V, B)}$ and $\Gamma_{(V, B)}$, along with parts (iiii) and (iv), now immediately imply that $\Gamma_{\left(V_{\lambda}, B_{\lambda}\right)}^{-1} \circ \Theta_{\left(V_{\lambda}, B_{\lambda}\right)}\left(b_{\lambda}^{\text {low }}\right)=J T_{w_{0}}\left(b_{\lambda}^{\text {low }}\right)=q^{(\lambda, \lambda) / 2+(\lambda, \rho)} b_{\lambda}$, completing the proof of (国).

We also need the following construction of the $R$-matrix due to Kirillov-Reshetikhin and Levendorskii-Soibelman. Due to a different choice of conventions, our $T_{w_{0}}$ is $K_{H_{\rho}}^{-1} T_{w_{0}}^{-1}$ in those papers, so we have modified the statement accordingly. As with Theorem 7.7 this expression is written using the $h$-adic completion of 
$U_{h}(\mathfrak{g})$, but gives a well-defined action on $V \otimes W$ for any finite dimensional type 1 $U_{q}(\mathfrak{g})$-module.

Theorem 7.7 ([KR, Theorem 3], [LS, Theorem 1]). The standard universal $R$ matrix can be realized as

$$
R=\exp \left(h \sum_{i, j \in I}\left(B^{-1}\right)_{i j} H_{i} \otimes H_{j}\right)\left(T_{w_{0}}^{-1} \otimes T_{w_{0}}^{-1}\right) \Delta\left(T_{w_{0}}\right) .
$$

Corollary 7.8. $\left(T_{w_{0}}^{-1} \otimes T_{w_{0}}^{-1}\right) \Delta\left(T_{w_{0}}\right)=\prod_{\beta} \exp _{q_{\beta}}\left[\left(1-q_{\beta}^{-2}\right) E_{\beta} \otimes F_{\beta}\right]$,

where the product is over all the positive roots of $\mathfrak{g}$, and the order of the terms is such that $\beta_{r}$ appears to the left of $\beta_{s}$ if $r>s$.

Proof. This follows immediately from Theorems 3.3 and 7.7, since the action of $R$ on $V_{\lambda} \otimes V_{\mu}$ is invertible.

As discussed in [KT], the following is equivalent to Theorem 7.7

Corollary 7.9 (see $\left[\mathrm{KT}\right.$, Comment 7.3]). Let $X=J T_{w_{0}}$. Then

$$
R=\left(X^{-1} \otimes X^{-1}\right) \Delta(X) .
$$

Lemma 7.10. Fix type 1 finite dimensional $U_{q}(\mathfrak{g})$ representations with chosen global bases $(V, B)$ and $(W, C)$. The operator $\left.\left(\Gamma_{(V, B)} \otimes \Gamma_{(W, C)}\right) \Gamma_{(V \otimes W, A)}\right)^{-1}$ acts on $V \otimes W$ as the identity, where $A$ is the global basis of $V \otimes W$ constructed from $B$ and $C$ in Definition 6.6.

Proof. It suffices to consider the case when $V=V_{\lambda}$ and $W=V_{\mu}$ are irreducible. Set

$$
m^{\Gamma}:=\left(\Gamma_{\left(V_{\lambda}, B_{\lambda}\right)} \otimes \Gamma_{\left(V_{\mu}, B_{\mu}\right)}\right)\left(\Gamma_{\left(V_{\lambda} \otimes V_{\mu}, A\right)}\right)^{-1}: V_{\lambda} \otimes V_{\mu} \rightarrow V_{\lambda} \otimes V_{\mu} .
$$

We must show that $m^{\Gamma}$ is the identity. $C_{\Gamma}$ is a Hopf algebra automorphism of $U_{q}(\mathfrak{g})$, so, as in Section 4 it follows that $m^{\Gamma}$ is an automorphism of $U_{q}(\mathfrak{g})$ representations. In particular, $m^{\Gamma}$ preserves isotypic components of $V_{\lambda} \otimes V_{\mu}$ and acts on each subquotient $Q_{\lambda, \mu}^{\nu}$ (see Definition 6.4). It is sufficient to show that the action on $Q_{\lambda, \mu}^{\nu}$ is the identity for all $\nu$. In fact, it is sufficient to consider the action on the highest weight space of $Q_{\lambda, \mu}^{\nu}$, since this generates $Q_{\lambda, \mu}^{\nu}$. This highest weight space has a basis consisting of $\left\{\overline{\overline{b_{\lambda} \otimes b}}: b \in S_{\lambda, \mu}^{\nu}\right\}$, where $S_{\lambda, \mu}^{\nu}$ is as in Definition 5.7 and we use the notation $\overline{\overline{a \otimes b}}$ to denote the image of $a \otimes b$ in $Q_{\lambda, \mu}^{\nu}$.

By Lemma 7.6 part (ii) and Corollary [7.8, we get

$$
\begin{aligned}
m^{\Gamma} & =\left(\operatorname{bar}_{\left(V_{\lambda}, B_{\lambda}\right)} \otimes \operatorname{bar}_{\left(V_{\mu}, B_{\mu}\right)}\right)\left(T_{w_{0}}^{-1} \otimes T_{w_{0}}^{-1}\right) \Delta\left(T_{w_{0}}\right) \operatorname{bar}_{\left(V_{\lambda} \otimes V_{\mu}, A\right)} \\
& =\left(\operatorname{bar}_{\left(V_{\lambda}, B_{\lambda}\right)} \otimes \operatorname{bar}_{\left(V_{\mu}, B_{\mu}\right)}\right) \prod_{\beta} \exp _{q_{\beta}}\left[\left(1-q_{\beta}^{-2}\right) E_{\beta} \otimes F_{\beta}\right] \operatorname{bar}_{\left(V_{\lambda} \otimes V_{\mu}, A\right)} .
\end{aligned}
$$

For convenience, set

$$
\Psi:=\left(\operatorname{bar}_{\left(V_{\lambda}, B_{\lambda}\right)} \otimes \operatorname{bar}_{\left(V_{\mu}, B_{\mu}\right)}\right) \prod_{\beta} \exp _{q_{\beta}}\left[\left(1-q_{\beta}^{-2}\right) E_{\beta} \otimes F_{\beta}\right] .
$$

Both $m^{\Gamma}$ and $\operatorname{bar}_{\left(V_{\lambda} \otimes V_{\mu}, A\right)}$ act in a well-defined way on each $Q_{\lambda, \mu}^{\nu}$, which implies that $\Psi$ does as well. 
The global basis $A$ was chosen so that $\operatorname{bar}_{\left(V_{\lambda} \otimes V_{\mu}, A\right)}\left(\overline{\overline{b_{\lambda} \otimes b}}\right)=\overline{\overline{b_{\lambda} \otimes b}}$ (see Definition 6.6). Since all $E_{\beta}$ kill $b_{\lambda}$ and $\left(\operatorname{bar}_{\left(V_{\lambda}, B_{\lambda}\right)} \otimes \operatorname{bar}_{\left(V_{\mu}, B_{\mu}\right)}\right)$ preserves $b_{\lambda} \otimes b$ by definition, we see that $\Psi\left(b_{\lambda} \otimes b\right)=b_{\lambda} \otimes b$, and, taking the image in $Q_{\lambda, \mu}^{\nu}$, $\Psi\left(\overline{\overline{b_{\lambda} \otimes b}}\right)=\overline{\overline{b_{\lambda} \otimes b}}$. Thus, using (20) , we see that $m^{\Gamma}$ acts on $\overline{\overline{b_{\lambda} \otimes b}}$ as the identity. The lemma follows.

Theorem 7.11. Fix type 1 finite dimensional $U_{q}(\mathfrak{g})$ representations with chosen global bases $(V, B)$ and $(W, C)$. Then $\left(\Theta_{(V, B)}^{-1} \otimes \Theta_{(W, C)}^{-1}\right) \Theta_{(V \otimes W, A)}$ acts on $V \otimes W$ as the standard $R$-matrix, where $A$ is the global basis of $V \otimes W$ constructed from $B$ and $C$ in Definition 6.6. This holds independently of the choices of global bases $B$ and $C$.

Proof. By Corollary 7.9 and Lemma 7.6(v), we get

$$
\begin{aligned}
R & =\left(\left(J T_{w_{0}}\right)^{-1} \otimes\left(J T_{w_{0}}\right)^{-1}\right) \Delta\left(J T_{w_{0}}\right) \\
& =\left(\Theta_{(V, B)}^{-1} \otimes \Theta_{(W, C)}^{-1}\right)\left(\Gamma_{(V, B)} \otimes \Gamma_{(W, C)}\right)\left(\Gamma_{(V \otimes W, A)}\right)^{-1} \Theta_{(V \otimes W, A)} .
\end{aligned}
$$

By Lemma 7.10, the expression $\left(\Gamma_{(V, B)} \otimes \Gamma_{(W, C)}\right)\left(\Gamma_{(V \otimes W, A)}\right)^{-1}$ acts as the identity.

Comment 7.12. By Theorem 7.11, the composition

$$
\left(\Theta_{(V, B)}^{-1} \otimes \Theta_{(W, C)}^{-1}\right) \Theta_{(V \otimes W, A)}
$$

does not depend on the choices on global bases $B$ and $C$. Introducing the notation $\Delta(\Theta)$ to mean $\Theta_{(V \otimes W, A)}$ and dropping the subscripts, we can interpret $\left(\Theta^{-1} \otimes\right.$ $\left.\Theta^{-1}\right) \Delta(\Theta)$ as (23) calculated using any global bases $B$ and $C$. Then Theorem 7.11 becomes (2) from the introduction. We also note that $\Theta_{(V, B)}$ is easily seen to be an involution, so the inverses in (23) are perhaps unnecessary.

\section{Future DiReCtions}

Although we have only proven Theorem 7.11 when $\mathfrak{g}$ is of finite type, much of the construction works in greater generality. We did not assume $\mathfrak{g}$ was a finite type in Section [6] so the expression $\left(\Theta_{(V, B)}^{-1} \otimes \Theta_{(W, C)}^{-1}\right) \Theta_{(V \otimes W, A)}$ makes sense for any symmetrizable Kac-Moody algebra. Since $C_{\Theta}$ is a coalgebra-antiautomorphism, the methods from Section 4 imply that

$$
\text { Flip } \circ\left(\Theta_{(V, B)}^{-1} \otimes \Theta_{(W, C)}^{-1}\right) \Theta_{(V \otimes W, A)}
$$

is an isomorphism of representations. Furthermore, it is true in general that (24) does not depend on the choice of $B$ and $C$. To see why, it is sufficient to consider the case when $V=V_{\lambda}$ and $W=V_{\mu}$ are irreducible. Then the global bases $B_{\lambda}$ and $B_{\mu}$ are unique up to multiplication by an overall scalar. It is straightforward to see that if $B_{\lambda}$ (or $B_{\mu}$ ) is scaled by a constant $z$, then $A$ is scaled by $z$ as well, and from there we see that both $\Theta_{\left(V_{\lambda}, B_{\lambda}\right)}$ and $\Theta_{\left(V_{\lambda} \otimes V_{\mu}, A\right)}$ are scaled by $z / \bar{z}$, where $\bar{z}$ is obtained from $z$ by inverting $q$. Thus the composition is unchanged.

As in Comment 7.12, we can now make sense of the expression $\left(\Theta^{-1} \otimes \Theta^{-1}\right) \Delta(\Theta)$ for all symmetrizable Kac-Moody algebras $\mathfrak{g}$. The fact that (24) defines an isomorphism is one of the properties required of a universal $R$-matrix. However, we have not proven the crucial equalities (5). Thus we ask:

Question 1. Is $\left(\Theta^{-1} \otimes \Theta^{-1}\right) \Delta(\Theta)$ a universal $R$-matrix for $U_{q}(\mathfrak{g})$ if $\mathfrak{g}$ is a general symmetrizable Kac-Moody algebra? If yes, is it the standard $R$-matrix? 


\section{ACKNOWLEDGEMENTS}

We thank Joel Kamnitzer, Noah Snyder, and Nicolai Reshetikhin for many helpful discussions.

\section{REFERENCES}

[CP] V. Chari and A. Pressley, A Guide to Quantum Groups, Cambridge University Press, 1994. MR1300632 (95j:17010)

[HK] A. Henriques and J. Kamnitzer, Crystals and coboundary categories, Duke Math. J., 132 (2006) no. 2, 191-216. MR 2219257 (2007m:17020)

$[\mathrm{KT}]$ J. Kamnitzer and P. Tingley. The crystal commutor and Drinfeld's unitarized $R$-matrix. J. Algebraic Combin. 29 (2009), no. 3, 315-335; arXiv:math/0707.2248v2. MR2496310

[K] M. Kashiwara, On crystal bases of the $q$-analogue of the universal enveloping algebras, Duke Math. J., 63 (1991), no. 2, 465-516. MR1115118 (93b:17045)

[KR] A. N. Kirillov and N. Reshetikhin, $q$-Weyl group and a multiplicative formula for universal R-matrices, Comm. Math. Phys. 134 (1990), no. 2, 421-431. MR1081014 (92c:17023)

[LS] S. Z. Levendorskii and Ya. S. Soibelman, The quantum Weyl group and a multiplicative formula for the R-matrix of a simple Lie algebra, Funct. Anal. Appl. 25 (1991), no. 2, 143-145. MR 1142216 (93a:17017)

[L] G. Lusztig. Introduction to quantum groups, Birkhäuser Boston Inc. 1993. MR,1227098 (94m:17016)

Massachusetts Institute of Technology, Department of Mathematics, 77 Massachusetts Avenue, Cambridge, Massachusetts 02139

E-mail address: ptingley@math.mit.edu 